\title{
Intrinsic magnetic relaxation in goethite
}

\author{
Quentin A. Pankhurst* \\ Davy-Faraday Research Laboratory, The Royal Institution of Great Britain, London WIS 4BS, United Kingdom and \\ Department of Physics, University College London, London WC1E 6BT, United Kingdom \\ Luis Fernández Barquín \\ Departamento CITIMAC, Facultad de Ciencias, Universidad de Cantabria, Santander 39005, Spain \\ James S. Lord \\ ISIS Facility, Rutherford Appleton Laboratory, Chilton, Didcot OX11 0QX, United Kingdom
}

Alex Amato and Ulrich Zimmermann

Laboratory for Muon Spin Spectroscopy, Paul Scherrer Institute, CH 5232 Villigen, Switzerland

(Received 5 January 2010; revised manuscript received 17 April 2012; published 29 May 2012)

\begin{abstract}
The intrinsic magnetic relaxation properties of goethite are elucidated via a comparative study of the magnetism of a stoichiometric and a nonstoichiometric sample, $\alpha$-FeOOH and $\alpha$ - $\mathrm{Fe}_{0.92} \square_{0.08} \mathrm{O}_{0.76} \mathrm{OH}_{0.24}$, where $\square$ denotes a vacancy site. It is proposed that both goethite samples exhibit "mode superparamagnetism" at low temperatures, wherein the iron moments undergo thermally induced fluctuations over an energy barrier separating two closely related geometrical arrangements for the sublattice spins. This is an intrinsic relaxation mechanism that was previously predicted from magnetic structure refinements, which we have identified here in ac susceptibility and thermal decay of remanence data. An additional magnetic relaxation mode occurs in the nonstoichiometric goethite. This mode is characterized by relatively slow fluctuations, observable on the microsecond timescale of muon spin relaxation experiments, but not on the fast timescale of Mössbauer spectroscopy, and appearing in ac susceptibility and dc magnetization data as blocking transitions in the range 70 to $100 \mathrm{~K}$. These data are best described as the result of "cluster ordering": the formation and relaxation of magnetic clusters in the disturbed environment of the nonstoichiometric lattice.
\end{abstract}

DOI: 10.1103/PhysRevB.85.174437

PACS number(s): 75.30.-m, 75.50.Ee, 75.75.Jn, 76.75.+i

\section{INTRODUCTION}

Goethite $(\alpha-\mathrm{FeOOH})$ is a naturally occurring antiferromagnetic mineral of orthorhombic structure and the space group Pnma. It is the most common of the $\mathrm{Fe}^{3+}$ oxyhydroxides and is widely found in rocks, soils, clays, and sediments. It originates both as a weathering product on iron oxide outcrops and as an intrinsic product of biogenic mineralization ${ }^{1}$ in bacteria, plants, insects, molluscs, fish, and mammals, including man. Its distinctive magnetic signature - an unusually high uniaxial anisotropy $\left(\mathrm{K}>10^{4} \mathrm{~J} / \mathrm{m}^{3}\right)$ coupled with an inherent weak ferromagnetic signal-has meant that it is used as an indicator of geological, environmental, and biological processes. These include palaeoclimatology evidence of a greenhouse effect on Earth 1.8 billion years ago, ${ }^{2}$ the impact on marine plankton of photochemical effects on iron colloids in ocean water, ${ }^{3}$ and a suggested role in human neurodegenerative diseases. ${ }^{4}$

Given the breadth of disciplines for which the magnetism of goethite is of fundamental importance, it is perhaps surprising that basic questions about the magnetism of goethite are still the subject of debate. This is in large part due to that fact that most naturally occurring goethite, and invariably all synthetic goethite, depart significantly from ideal stoichiometry, with vacancies and/or diamagnetic impurity atoms occupying $\mathrm{Fe}^{3+}$ sites in the lattice. This has led to the literature being dominated by the magnetism of nonstoichiometric goethite, in which the properties are determined by competing effects, including moment canting, magnetic relaxation, and spin clustering. These, in turn, are subjects that have only recently received full recognition as the result of research into the behavior of modern magnetic materials—such as the formation of cluster superspin glasses in strongly interacting nanogranular alloys, 5,6 the use of exchange-biased nanoparticles to beat the superparamagnetic limit in nanoparticulate magnetic recording, ${ }^{7}$ the appearance of an exchange-mediated slowing of magnetic dynamics in aggregated antiferromagnetic nanoparticles, ${ }^{8}$ or the onset of cluster magnetism in the phase-separated manganite oxides..$^{9,10}$ In addition to this, the understanding of the core and shell properties in magnetic nanoparticles is of great importance. ${ }^{11,12}$ If the core of the magnetic particles is ferromagnetic, the role of the surface layer is masked. On the contrary, if antiferromagnetic particles were used (as goethite is), the magnetic response of surface atoms would be more evident. ${ }^{13}$

Another factor has been that the bulk magnetic properties of goethite do not lend themselves to facile interpretation. Although it shows antiferromagnetism, it also possesses a weak ferromagnetic moment, the strength of which is highly sample dependent. Various explanations for this moment have been put forward over the years, including finite size effects, ${ }^{14,15}$ unequal iron sublattice occupancies, ${ }^{16}$ and uncompensated surface spins. ${ }^{17}$ It was not until 1995 that Coey et al. performed a neutron diffraction study on a high-purity, natural, single-crystal goethite and by means of a detailed magnetic Rietveld refinement showed that the weak moment is the result of an intrinsically canted antiferromagnetic structure along the crystallographic $b$ axis. ${ }^{18}$ This allows a 
degenerate behavior, with intrinsic fluctuations of the magnetic component. ${ }^{18}$ In short the fluctuations over a feeble anisotropy barrier are connected to the axial component of the sublattice magnetization. Similar experiments have not yet been reported for nonstoichiometric goethite.

Instead, and for the most part, discussion of the magnetic properties of both natural and synthetic goethite has relied, to a large extent, on ${ }^{57} \mathrm{Fe}$ Mössbauer effect data. In principle, Mössbauer spectroscopy is well suited to studying antiferromagnets, since it gives a direct measure of the sublattice magnetization, but in practice it is somewhat open to interpretation.

Early Mössbauer studies showed conclusively the Néel transition in goethite. ${ }^{19,20}$ However, subsequent studies revealed sample-dependent behavior, with fine-particle goethite typically having asymmetrically broadened absorption lines at low temperature, collapsing to a doublet at higher temperatures, usually in the range $330-400 \mathrm{~K}$. The origin of this line broadening became the focus of much debate. Initially, it was attributed to superparamagnetism (SPM), a nanoscale phenomenon in which the anisotropy energy of small volume particles becomes comparable to the thermal activation energy, and the atomic moments cooperatively undergo reversals in direction. ${ }^{21,22}$ It was later argued that the behavior was not that of a simple superparamagnet, and the mean-field concept of SPM was introduced to account for the effect of interparticle interactions and to explain certain features of the Mössbauer data in applied fields. ${ }^{23}$ There, the basic concept, which overall considers the collective magnetic behavior of the particles, is that the interparticle interactions (commonly due to exchange coupling and/or dipolar interaction) promote magnetic coupling involving a superferromagnetic (SFM) ordering of the particle magnetization at temperatures higher than the expected blocking temperature; that is, in the state in which they should exhibit fast relaxation. The model does not necessarily imply a macroscopic ferromagnetic moment for the sample. An extension of this concept has been applied for metallic systems and multilayers, with a special attention to magnetic dynamics, to express (again) the coupling among magnetic particles, which are ferromagnetically coupled forming effectively a domain in a classical sense. ${ }^{6}$ There, it was also argued the need of intercalated particles promoting the superferromagnetic order. In fact, the concept of a strong effective superferromagnetic coupling among particles has been revisited very recently to explain the thermal variation of the hyperfine field in as-prepared and annealed $\left(150^{\circ} \mathrm{C}, 24 \mathrm{~h}\right)$ goethite samples comprising rodlike entities bundled together $(5 \times 50 \mathrm{~nm})^{24}$ and in ball-milled compounds, which were also the object of a high-resolution transmission electron study. ${ }^{25}$

However, in 1992 Bocquet et al. called into question both of these inherently dynamic explanations for the Mössbauer line broadening in goethite. ${ }^{26}$ By conducting a combined neutron diffraction, Mössbauer spectroscopy, and magnetization study on a single fine-particle goethite sample, Bocquet et al. found that all three techniques showed discontinuous magnetic features at the same temperature. Given that the characteristic measurement times of the three techniques covered seven orders of magnitude, these data indicated a magnetic ordering transition rather than SPM. A subtle variation of the SFM idea was proposed, namely, cluster ordering. ${ }^{27}$ In this model it was predicted that reduced Néel temperatures would occur due to the collective magnetic ordering of clusters of $\mathrm{Fe}^{3+}$ moments, the clusters, in turn, arising from the percolation segregation of $\mathrm{Fe}^{3+}$ sublattice spins by vacancy defects. This model was found to work well with a wide range of natural and synthetic samples. ${ }^{27}$ Anyhow, the cluster ordering model has not been universally accepted, and it has been argued that the superferromagnetism model is more physically reasonable. $^{24,25}$

It should be stressed, and as a corollary to the neutron diffraction experiment commented above, ${ }^{18}$ that mode superparamagnetism (MSPM) is a consistent model in which the dynamic effects arise from thermally activated transverse spin fluctuations between two equal energy states in the canted moment structure. It is then very similar conceptually to conventional SPM, except that in MSPM it is the transverse components of the spins normal to the primary axis of collinearity (in goethite the $b$ axis) that are flipping between up-down and down-up states. It is, perhaps, surprising that this seemingly intuitive concept has not been explored in more detail since it was first proposed. One reason might be that in the original work ${ }^{18}$ the phenomenon was proposed as the explanation for the observed Mössbauer line broadening in goethite, a suggestion that was strongly refuted by others on the grounds of the magnitude of the effect it could support. ${ }^{28}$ Nevertheless, it remains clear that in appropriate systemsand goethite is undoubtedly a good candidate-MSPM is a phenomenon that may be present and may be observable, given the right experimental conditions.

The present work is an attempt to move on from previous studies and to offer a conclusive contribution on the magnetism of goethite: (1) by making measurements on two representative samples, one a high-purity stoichiometric natural goethite, the other a well-characterized nonstoichiometric synthetic goethite; (2) by making measurements on timescales covering many orders of magnitude, from nanoseconds to tens of seconds, and unifying the understanding thereby gained; and (3) by making muon spin relaxation $(\mu \mathrm{SR})$ measurements on goethite - a technique with an intermediate (ca. microsecond) timescale that transpires to be very helpful when it comes to distinguishing between the various models of relaxation behavior.

\section{EXPERIMENTAL}

The stoichiometric sample was a high-purity natural goethite mined from the Harz Mountains in Germany and supplied to us by E. De Grave of the State University of Ghent, Belgium. The nonstoichiometric sample was a synthetic goethite denoted L20h, supplied by S. Bocquet, formerly of Monash University, Australia. Thermogravimetric analysis (TGA) measurements were made on a PerkinElmer TGA7, at a heating rate of $20 \mathrm{~K} \mathrm{~min}^{-1}$. Samples of ca. 20-mg mass were placed in open platinum pans in an atmosphere of flowing nitrogen (flow rate $60 \mathrm{ml}$ $\min ^{-1}$ ) that reduced the likelihood of oxidation. The ${ }^{57} \mathrm{Fe}$ Mössbauer spectra were collected on a Wissel MA-260S constant acceleration spectrometer, calibrated against $\alpha$-iron at room temperature. A triangular drive waveform was used, and the spectra were folded to remove baseline curvature. 
The spectra were least-squares fitted as a superposition of Lorentzian sextets. dc magnetization data were recorded using a Quantum Design magnetic property measurement system (MPMS) superconducting quantum interface device (SQUID) magnetometer operated under zero-field-cooled (ZFC) and field-cooled (FC) sequences. The sample temperature was changed at ca. $3 \mathrm{~K} \mathrm{~min}^{-1}$ and stabilized at each measuring point for approximately $2 \mathrm{~min}$. Each data point was averaged over three 30-s instrument scans, and the total measurement time was ca. $100 \mathrm{~s}$. ac susceptibility measurements were made using a Quantum Design Physical Property Measurement System (PPMS). Both in-phase $\left(\chi^{\prime}\right)$ and out-of-phase $\left(\chi^{\prime \prime}\right)$ data were recorded using an excitation field of $h=10 \mathrm{Oe}$ at frequencies $v=100 \mathrm{~Hz}$ to $10 \mathrm{kHz}$. The $\mu \mathrm{SR}$ experiments were performed on the MuSR spectrometer at the ISIS facility at the Rutherford Appleton Laboratory, United Kingdom, and on the GPS and Dolly spectrometers at the Paul Scherrer Institute, Switzerland. The $\mu$ SR spectra were analyzed using the WiMDA program. ${ }^{29}$

\section{SAMPLE CHARACTERIZATION}

Both the Harz and L20h samples have been the subject of earlier studies. ${ }^{26,27,30}$ Both have narrow particle size distributions, with mean particle dimensions of $700 \times 450 \times 450 \mathrm{~nm}^{3}$ for the Harz sample, and $50 \times 12 \times 6 \mathrm{~nm}^{3}$ for L20h. The Harz goethite is a high-purity, well-crystallized, bulk material, with an aluminum content of less than 0.8 at.\% and a Néel temperature, $\mathrm{T}_{\mathrm{N}} \approx 398 \mathrm{~K}$, which is close to the $400 \mathrm{~K}$ seen in the best goethite. ${ }^{31}$ The L20h goethite is a fine-particle material that was prepared by chemical precipitation. ${ }^{26}$ It has a reduced $\mathrm{T}_{\mathrm{N}}$ of ca. $358 \mathrm{~K}$ due to iron vacancies in the lattice. ${ }^{32}$ The concentration of vacancies can be estimated from the limiting slope $T_{N}^{-1} d T_{N} / d p=1.37$ for a simple cubic lattice, ${ }^{33}$ where $p$ is the $\mathrm{Fe}^{3+}$ ion concentration in the lattice. Taking the bulk $T_{N}=400 \mathrm{~K}$ yields $p \approx 1.00$ (no vacancies) for the Harz goethite and 0.92 (8\% vacancies) for L20h.

Further information on the composition of the samples can be obtained via thermal analysis (Fig. 1), wherein the phase transformation on heating is taken to be

$$
2 \mathrm{Fe}_{1-\mathrm{x}} \square_{\mathrm{x}} \mathrm{OOH} \rightarrow(1-\mathrm{x}) \mathrm{Fe}_{2} \mathrm{O}_{3}+\mathrm{H}_{2} \mathrm{O}+1.5 \mathrm{xO}_{2},
$$

and $\square$ denotes a vacancy site. The weight loss between $120^{\circ} \mathrm{C}$ and $500{ }^{\circ} \mathrm{C}$ for the Harz sample is ca. $9.7 \%$, which is slightly less than the $10.1 \%$ expected for pure goethite. The explanation

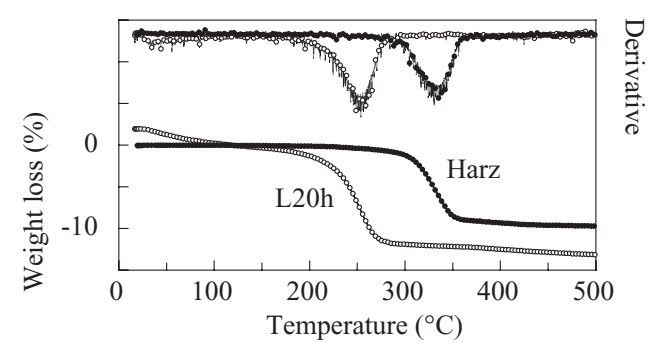

FIG. 1. TGA data for a stoichiometric goethite, Harz, and a nonstoichiometric goethite containing $8 \%$ vacancies on the $\mathrm{Fe}^{3+}$ lattice, L20h. The lower curves show weight loss as a function of temperature, and the upper curves are their derivatives with respect to temperature. for this lies in a previously documented ${ }^{30}$ trace hematite impurity in the sample. The precise composition is thus

$$
\mathrm{Harz}=\alpha-\mathrm{FeOOH}+0.025 \alpha-\mathrm{Fe}_{2} \mathrm{O}_{3} .
$$

For L20h there is some weight loss below $100^{\circ} \mathrm{C}$, which can be attributed to extra-framework encapsulated water. Such an encapsulation is to be expected in a chemically synthesized material. The weight loss between $120^{\circ} \mathrm{C}$ and $500^{\circ} \mathrm{C}$ is ca. $13.1 \%$, which from Eq. (1) corresponds to a vacancy concentration of $\mathrm{x}=0.08$, in excellent agreement with the figure obtained from $\mathrm{T}_{\mathrm{N}}$. We deduce that

$$
\mathrm{L} 20 \mathrm{~h}=\alpha-\mathrm{Fe}_{0.92} \square_{0.08} \mathrm{O}_{0.76} \mathrm{OH}_{0.24}+0.05 \mathrm{H}_{2} \mathrm{O}
$$

is the composition, maintaining the chemical neutrality. ${ }^{34}$ Although the samples present inconveniences with respect to single crystals of goethite, they offer the advantage of being part of a series of carefully synthesized/selected goethite samples with different particle sizes, as reported previously. ${ }^{27}$ In the following, the magnetic influence of the particular hematite impurity will be discussed when pertinent.

\section{MÖSSBAUER SPECTROSCOPY}

The Mössbauer data in Fig. 2 were recorded for comparison with previous Mössbauer studies of bulk and fine-particle goethite and to probe for nanosecond timescale dynamic phenomena, as well as to verify the presence of the hematite impurity in the Harz sample.

The zero-field room-temperature Mössbauer spectra (open symbols in Fig. 2) are identical to those recorded previously by others. ${ }^{26,30}$ The Harz spectrum is dominated by a magnetically split sextet, with narrow lines and hyperfine parameters (see Table I) that are typical of well-crystallized goethite. The hematite impurity is visible as a minor sextet component with outermost lines at ca. $\pm 8.3 \mathrm{mms}^{-1}$ and has hyperfine parameters typical of well-crystallized hematite. Its relative spectral/atomic weighting, assuming equal recoil-free fractions, is ca. 3 at.\% Fe, in good agreement with the 5 at.\% derived from Eq. (2).

No impurities are present in the L20h spectrum, but the absorption lines are broader than in Harz and are asymmetric, with the narrower part of each line at the higher absolute

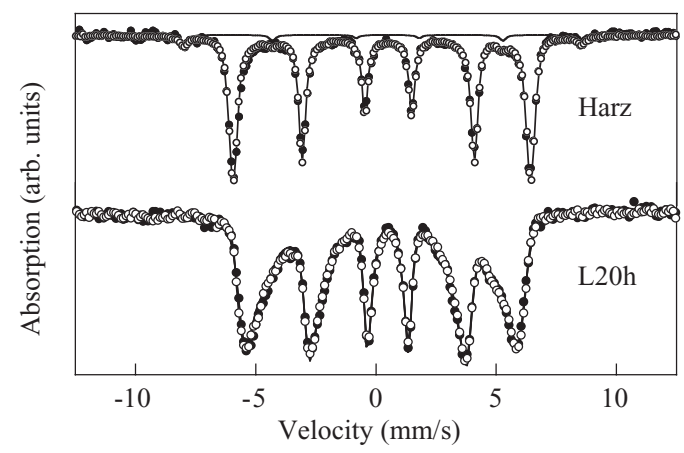

FIG. 2. Superposition of ${ }^{57} \mathrm{Fe}$ Mössbauer spectra measured at room temperature in zero field (o) and in $11 \mathrm{kOe}$ perpendicular to the $\gamma$-ray beam ( $\bullet$ ) for Harz and L20h goethite. For the Harz sample the presence of a small impurity phase of hematite indicated by a solid line showing its associated subspectrum. 
TABLE I. Parameters derived from the room-temperature Mössbauer spectra of Fig. 2: isomer shift $\delta$, quadrupole shift $2 \varepsilon$, relative absorption Area, mean hyperfine field $\langle H\rangle$, and standard deviation $\sigma_{H}$. The magnetic sextets were modeled as Gaussian distributions of elementary Lorentzian sextets, each with a linewidth of $\Gamma=0.12 \mathrm{mms}^{-1}$. The number in parentheses is the uncertainty in the last digit.

\begin{tabular}{|c|c|c|c|c|c|}
\hline Sample & $\delta\left(\mathrm{mms}^{-1}\right)$ & $2 \varepsilon\left(\mathrm{mms}^{-1}\right)$ & Area $(\%)$ & $\langle H\rangle(\mathrm{kOe})$ & $\sigma_{H}(\mathrm{kOe})$ \\
\hline \multirow[t]{2}{*}{ Harz in zero field } & $0.34(1)$ & $-0.26(1)$ & $72.2(3), 24.5(3)$ & $383(1), 320(7)$ & $7(1), 133(6)$ \\
\hline & $0.38(1)$ & $-0.21(1)$ & $3.3(3)$ & $513(1)$ & $6(2)$ \\
\hline L20h in zero field & $0.36(1)$ & $-0.27(1)$ & $29(5), 37(5), 34(5)$ & $256(20), 315(12), 353(2)$ & $73(7), 30(9), 14(2)$ \\
\hline \multirow[t]{2}{*}{ Harz in $11 \mathrm{kOe}$} & $0.35(1)$ & $-0.27(1)$ & $71.8(3), 25.1(3)$ & $382(3), 325(2)$ & $10(4), 88(13)$ \\
\hline & $0.36(1)$ & $-0.18(1)$ & $3.1(3)$ & $513(1)$ & $1(1)$ \\
\hline $\mathrm{L} 20 \mathrm{~h}$ in $11 \mathrm{kOe}$ & $0.38(1)$ & $-0.26(1)$ & $34(5), 35(5), 31(5)$ & $263(13), 316(11), 353(1)$ & $80(5), 30(8), 15(2)$ \\
\hline Goethite standard ${ }^{32}$ & 0.37 & -0.26 & - & 380 & - \\
\hline Hematite standard ${ }^{32}$ & 0.37 & -0.20 & - & 518 & - \\
\hline
\end{tabular}

velocity for that line, and the high-absolute-velocity onset of each line coinciding with the absorption maxima in Harz.

Such an asymmetrically broadened spectrum is typical of many recorded in fine-particle goethite since the 1960s. There has been much debate about the origin of the broadening, ${ }^{27}$ but the current consensus favors a static distribution of hyperfine fields due to a distribution in the local environments of the $\mathrm{Fe}^{3+}$ ions, which, in turn, is due to the iron-site vacancies and defects normally found in fine-particle goethite. An alternative is that the asymmetry is a reflection of dynamic effects such as SPM, causing the Fe moments to fluctuate on a timescale comparable to the $\tau_{\mathrm{m}} \approx 2 \mathrm{~ns}$ measurement time of the room-temperature Mössbauer spectrum of goethite. However, it has been argued ${ }^{27}$ that this hypothesis cannot be true because the uniaxial magnetic anisotropy energies required for the supposed blocking transitions are too small: $\mathrm{K}$ in the range $0.4-1.8 \mathrm{kJm}^{-3}$, whereas current estimates for pure goethite put $\mathrm{K}$ closer to $30 \mathrm{kJm}^{-3}$ at room temperature and $60 \mathrm{kJm}^{-3}$ at $4.2 \mathrm{~K}^{18}$

The applied field spectra in Fig. 2 (closed symbols) show no change between the zero-field and the applied field measurements, as evidenced by the superposition of the data, and are not enough to get a clear cut test of whether the broadening is a static or a dynamic effect. Furthermore, there exists near-perfect superposition of the zero-field and 11-kOe spectra in both the Harz and L20h goethite, especially in the region of the second and fifth lines of the sextet-the relative intensities of which can be a sensitive probe of moment reorientation. ${ }^{35}$

\section{MAGNETOMETRY}

To probe the magnetic properties at a longer timescale, a series of magnetometry experiments (with characteristic measurement times $\tau_{\mathrm{m}} \approx 100 \mathrm{~s}$ ) were recorded; viz. the magnetic hysteresis curves in Fig. 3, the ZFC-FC curves in Fig. 4, and the thermal decay of remanence (TDR) curves in Fig. 5.

The hysteresis curves at $10 \mathrm{~K}$ (Fig. 3) are dominated by the linear characteristic of an antiferromagnetic powder in field much less than its magnetocrystalline anisotropy field. L20h has a larger dc susceptibility (i.e., slope) than does Harz, which implies that its anisotropy is smaller than in Harz. Close inspection reveals that in both samples the hysteresis curves are open to at least $\pm 40 \mathrm{kOe}$, as seen by subtracting the decreasing field branch from the increasing field branch (lower inset, Fig. 3). Open loops are a known feature of the high anisotropy of goethite. ${ }^{18}$ The smaller difference between the branches in Harz compared to L20h is a sign of its stoichiometric structure, without the vacancies and associated pinning centers of L20h. Averaging the branches of the Harz loop and subtracting a linear curve reveals the sigmoidal curve, which shows the presence of magnetization shape close to that of a ferromagnet. This very minor signal is surely due to the presence of some uncompensated moments (upper inset, Fig. 3), which reaches magnetic saturation at ca. $\pm 5 \mathrm{kOe}$, often present in goethite samples. The detected presence of hematite, below the Morin transition, is thus masked by the majority signal from the goethite crystals at $10 \mathrm{~K}$.

ZFC-FC magnetometry curves (Fig. 4) show a distinct difference in behavior between the two samples. L20h has a larger magnetization at all temperatures and a more pronounced difference between ZFC and FC branches, than does Harz. There is also a peak in the ZFC magnetization of L20h (at $\mathrm{T}_{\text {peak }} \approx 40 \mathrm{~K}$ for the $\mathrm{H}=10 \mathrm{kOe}$ data shown in Fig. 4) that does not appear in any of the other curves. The existence of similar ZFC peaks is well documented in fine-particle

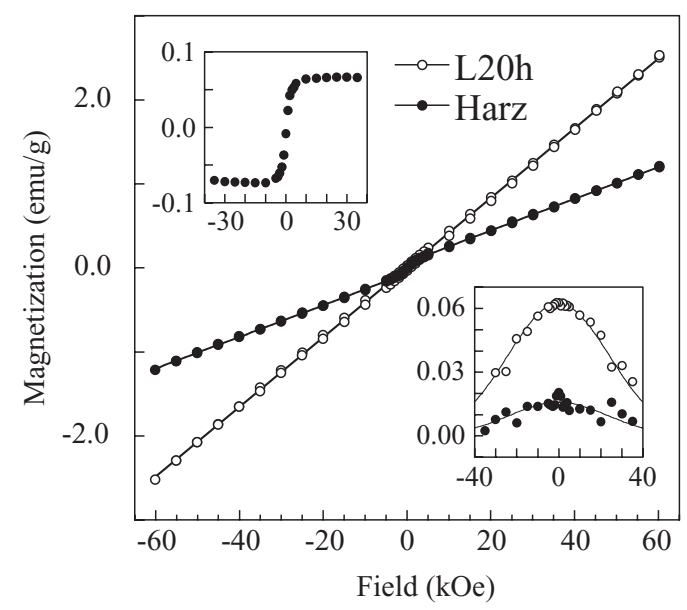

FIG. 3. Hysteresis loops at $10 \mathrm{~K}$ for the Harz and L20h goethite sample. The lower-right inset shows the difference $\Delta \mathrm{M}=\mathrm{M}_{+}-\mathrm{M}_{-}$ between the increasing and decreasing field branches of the loops, which is nonzero, and indicates a degree of intrinsic hysteresis. The upper-left inset shows the average of the $\mathrm{M}_{+}$and $\mathrm{M}_{-}$branches of the Harz data, after subtracting a linear slope. 


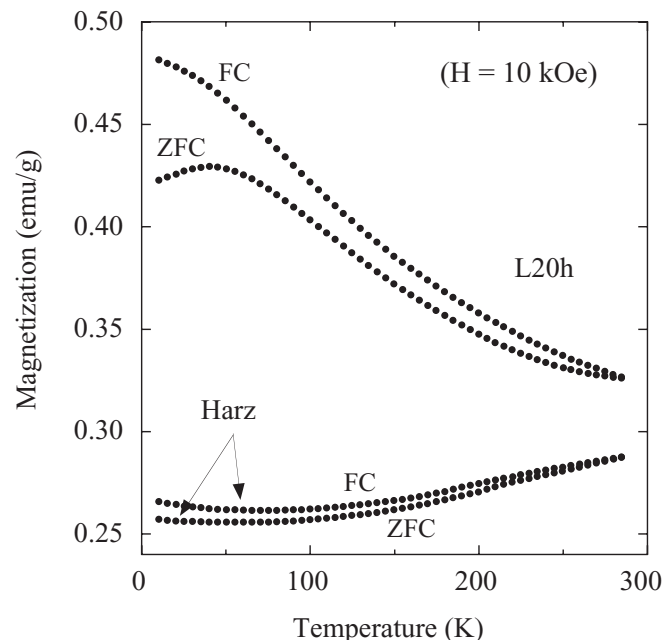

FIG. 4. ZFC and FC magnetization curves for Harz and L20h goethite measured as a function of temperature in an applied field of $\mathrm{H}=10 \mathrm{kOe}$ after having been cooled to $10 \mathrm{~K}$ in either zero field (ZFC) or $\mathrm{H}=10 \mathrm{kOe}(\mathrm{FC})$. Data were also recorded for smaller fields, with the observed ZFC-FC data qualitatively resembling the shown curves. $T_{\text {peak }}$ marks the temperature at which a peak is evident in the ZFC data for the L20h sample. The peak in the ZFC shifts down in temperature with the increase of $\mathrm{H}$.

iron oxides and is usually taken as a sign of magnetic blocking/unblocking leading to superparamagnetic behavior at higher temperatures. ${ }^{36}$ The persistence of the L20h ZFC peak even in $\mathrm{H}=10 \mathrm{kOe}$ indicates a strongly anisotropic local mechanism, quite different from, for example, the situation in canonical spin glasses, where fields of a few tens of oersted are enough to suppress the divergence of the ZFC and FC branches. ${ }^{37}$ The $\mathrm{T}_{\text {peak }}$ increases as field decreases, reaching ca. $70 \mathrm{~K}$ for $\mathrm{H}=10 \mathrm{Oe}\left(\mathrm{T}_{\text {peak }}=68 \mathrm{~K}\right.$ at $\left.\mathrm{H}=500 \mathrm{Oe}\right)$, which is usual in superparamagnets and spin-disordered systems, for example, as reported in $\gamma-\mathrm{Fe}_{2} \mathrm{O}_{3}$ fine particles, where it was associated with a surface disordered magnetic structure, ${ }^{37}$ having previously been theoretically predicted as an indicator of collective blocking due to interparticle interactions. ${ }^{38}$ Although goethite is an antiferromagnet with

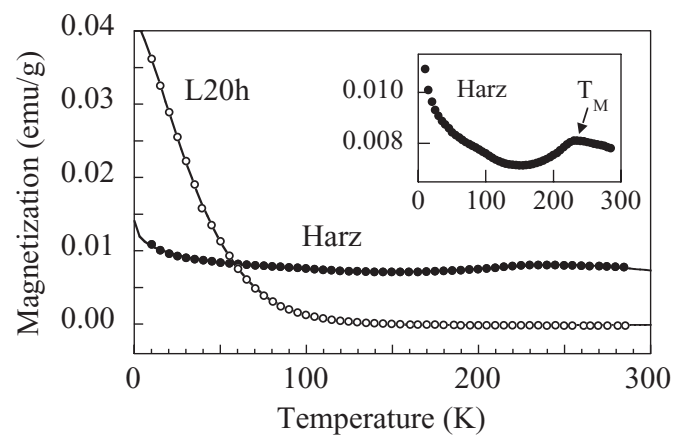

FIG. 5. TDR curves for Harz and L20h goethite, recorded in zero field after $60 \mathrm{kOe}$ had been applied and removed at $10 \mathrm{~K}$. The solid lines are fits to phenomenological models, as discussed in the text. The inset is an expanded plot of the Harz data that highlights the contribution of the small hematite impurity; $\mathrm{T}_{\mathrm{M}}$ marks the Morin transition temperature in hematite. very weak dipolar interactions, the magnetic coupling through the boundaries and the documented weak ferromagnetism in the goethite could allow such a collective transition.

Also notable is the persistence of the ZFC-FC branching for both samples, which implies it is not a dynamic effect, but instead something intrinsic to goethite. We attribute it to the locking in, in the ZFC state, of the Fe moments in a random distribution of sublattice magnetization canting directions, as opposed to the FC case where more favored canting axis directions are chosen so as to minimize the field energy associated with the applied field. In the stoichiometric sample this effect is relatively small, as it relies on the intrinsic noncollinearity of the spins (as revealed by neutron diffraction data), ${ }^{18}$ while in the nonstoichiometric sample it is more pronounced due to the presence of magnetic clusters.

Corroborating information on this can be deduced from TDR data obtained by applying and removing a field of $60 \mathrm{kOe}$ at $10 \mathrm{~K}$ and measuring the magnetization, in zero field, on heating to $285 \mathrm{~K}$ (Fig. 5). There is a clear drop in the remanence as temperature is increased in $\mathrm{L} 20 \mathrm{~h}$, which is also visible, albeit less pronounced, in Harz. This is consistent with a temperaturedriven return to a net zero magnetization state following the freezing in of a preferred canting state at low temperature in the presence of the large applied field. The Harz data is somewhat complicated by the presence of the hematite impurity, which contributes a distinctive remanence signal that includes a peak at ca. $250 \mathrm{~K}$ associated with the Morin phase transition in hematite.

Phenomenological fits to the data are consistent with there being a common origin to the decay in remanence in both samples. The L20h data was modeled as a power exponential of the form $\alpha \exp \left(\beta \mathrm{T}^{\gamma}\right)$, with $\beta \approx 0.005 \mathrm{~K}^{-\gamma}$ and $\gamma \approx$ 1.4. The characteristic temperature for this decay, when the function equaled $\alpha / \mathrm{e}$, was $42 \pm 3 \mathrm{~K}$. The Harz remanence was fitted as a power exponential plus a Gaussian centered at $250 \mathrm{~K}$ for the hematite. The exponent $\gamma$ was ill defined and was fixed to be 0.5 . Nevertheless the $\alpha / \mathrm{e}$ characteristic was estimated to be $30 \pm 10 \mathrm{~K}$, which is close enough to the L20h figure to imply common and intrinsic phenomena. It is suggestive of the predicted low-temperature effect of MSPM, with the thermally activated transitions between near-degenerate states in the canted moment structure.

\section{AC SUSCEPTIBILITY}

Alternating current susceptibility data provide more direct information on the moment dynamics. Figure 6 shows the in-phase susceptibility $\chi^{\prime}(\mathrm{T})$ at frequencies $v=100 \mathrm{~Hz}$ and $10 \mathrm{kHz}$, corresponding to measurement times of $10 \mathrm{~ms}$ and $100 \mu \mathrm{s}$, respectively. The shapes of the curves broadly reflect those seen in the ZFC-FC magnetization data.

For the stoichiometric goethite, Harz, the data show (1) a linear increase in $\chi^{\prime}$ with increasing temperature, which is the canonical behavior of an antiferromagnet below its Néel temperature; and (2) a strong upturn in $\chi^{\prime}$, only visible in a low-temperature range, the origin of which we tentatively ascribe to MSPM. An alternative explanation for the latter could be a Curie-law contribution from occasional isolated $\mathrm{Fe}$ atoms in the lattice. In fact the data can be fitted quite well to a phenomenological model of the sum of Curie-law and linear 


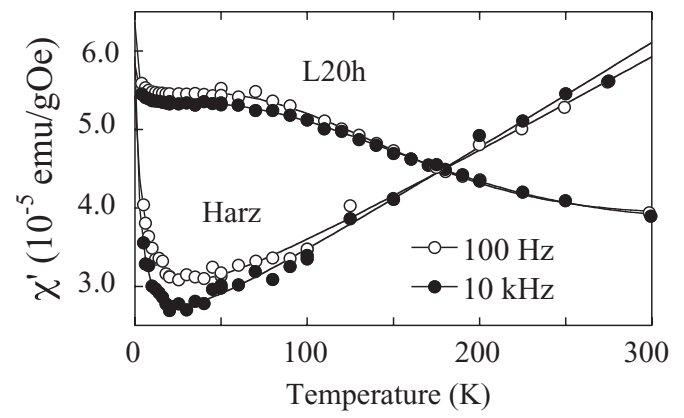

FIG. 6. ac susceptibility data for L20h goethite, measured with an excitation field of $10 \mathrm{Oe}$, in zero external field. A low-temperature upturn is clearly visible for both samples.

terms, $\chi^{\prime}(\mathrm{T})=\alpha /(\mathrm{T}+\beta)+k \mathrm{~T}$, with $\beta \approx 2 \mathrm{~K}$. This is plotted as the solid lines in Fig. 6. However, similar low-temperature upturns in $\chi^{\prime}$ have been reported in other near-stoichiometric goethite, including one from Gangaol, Burkina Faso, ${ }^{39}$ for which $\mathrm{T}_{\mathrm{N}}=380 \mathrm{~K}$, which implies that it is an intrinsic effect; and further evidence below leads us to conclude that this is not simply a paramagnetic relaxation signal.

For the nonstoichiometric goethite, L20h, the $\chi^{\prime}(\mathrm{T})$ data are dominated by a Gaussian-like term, reminiscent of that commonly seen in superparamagnets, although there is in addition a distinct upturn in $\chi^{\prime}$ below $20 \mathrm{~K}$. To explore these features in more detail, a more comprehensive dataset was recorded on $\mathrm{L} 20 \mathrm{~h}$ at $v=300 \mathrm{~Hz}$ and at 1,3 , and $10 \mathrm{kHz}$ from $8 \mathrm{~K}$ to room temperature (see Fig. 7). Additional data were obtained at $1 \mathrm{kHz}$ from room temperature to $400 \mathrm{~K}$, although as it was known from the earlier TGA experiments that this was a semidestructive measurement in which water was driven from the sample; it was only done once, and thereafter that particular aliquot was discarded.

The L20h $\chi^{\prime}(\mathrm{T})$ data in Fig. 7 show three distinct features: (1) below $20 \mathrm{~K}$, the same Curie-like upturn as in Harz, albeit that it is frequency dependent, being most pronounced for $v \leqslant 3 \mathrm{kHz}$, and apparently absent for $v=10 \mathrm{kHz}$; (2) a peak centered at ca. $60-70 \mathrm{~K}$, which broadens and shifts to higher

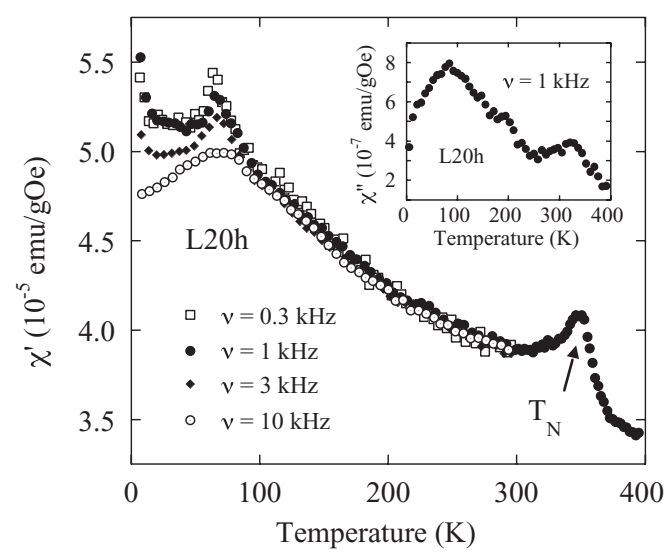

FIG. 7. ac susceptibility data for L20h goethite, measured with an excitation field of $10 \mathrm{Oe}$, in zero external (biasing) field. The definition of the Néel temperature is excellent (marked with an arrow). Inset: L20H complex component with two maxima in the vicinity of cluster coupling and Néel temperature. temperature with increasing $v$; and (3) recorded at $1 \mathrm{kHz}$ only, a second peak at ca. $348 \mathrm{~K}$. The two peaks, but not the low-temperature upturn, are also seen in the out-of-phase susceptibility, $\chi^{\prime \prime}(\mathrm{T})$, as shown in the inset in Fig. 7.

The frequency dependence of the low-temperature upturn in $\chi^{\prime}$ is a definitive indication that it is not a Curie-law effect due to isolated $\mathrm{Fe}$ atoms in the lattice. If its origin were paramagnetic, then it should have been observable equally well at a measurement timescale of $100 \mu \mathrm{s}$ (corresponding to $v=10 \mathrm{kHz})$ as at $3.3 \mathrm{~ms}(v=300 \mathrm{~Hz})$. Instead, it is clear that $100 \mu \mathrm{s}$ is too fast to see the effect. As such, the upturn, which is observed in both the Harz and L20h samples, must be an intrinsic magnetic relaxation phenomenon, and is most likely to be the predicted MSPM.

The frequency-dependent peaks at $60-70 \mathrm{~K}$ in $\mathrm{L} 20 \mathrm{~h}$ indicate that additional magnetic relaxation is taking place. It is notable that this peak shifts to lower temperature as $v$ increases and the measurement time decreases, which is contrary to the expected increase in blocking temperature with measurement time that is expected in canonical SPM. On inspection the frequency dependence of the peak temperature (not shown) was found to follow reasonably well a phenomenological Vogel-Fulcher law. Such a behavior is often taken as evidence of magnetically interacting clusters or particles, ${ }^{40}$ and in the context of the models of relaxation behavior already proposed for goethite, may best be explained as due to "cluster ordering." 25,27

The peak in $\mathrm{L} 20 \mathrm{~h}$ at $\mathrm{T}_{\mathrm{N}} \approx 348 \mathrm{~K}$ is undoubtedly due to the antiferromagnet-to-paramagnet Néel transition in goethite. It is somewhat lower than the $\mathrm{T}_{\mathrm{N}} \approx 358 \mathrm{~K}$ value derived from dc-magnetization in another batch of the same material in $1992,{ }^{26}$ which probably reflects a small relative change in the water content of the sample with storing time. ${ }^{41}$

\section{MUON SPIN RELAXATION}

In the experiments described above, evidence for magnetic relaxation was seen in TDR magnetometry data, where the measurement timescale $\tau_{\mathrm{m}} \approx 100 \mathrm{~s}$, and in ac susceptibility data, where $\tau_{\mathrm{m}}$ was in the range $10 \mathrm{~ms}$ to $100 \mu \mathrm{s}$. On the timescale of Mössbauer spectroscopy, no dynamic behavior was evident. It is, therefore, potentially valuable to probe the magnetic properties of the Harz and L20h goethite samples using the relatively uncommon modality of $\mu \mathrm{SR}$, a technique for which $\tau_{\mathrm{m}}$ is sample dependent, and of the order of tens to hundreds of nanoseconds. ${ }^{42}$ This enables the $\mu$ SR technique as another probe to check dynamics and magnetic relaxation, providing information on the SPM and or spin-glass behaviors in monodispersed nanometric $\gamma-\mathrm{Fe}_{2} \mathrm{O}_{3} .{ }^{43}$

In $\mu \mathrm{SR}$ experiments, spin-polarized muons are implanted into a sample, where they precess about any local field not parallel with their initial polarization. ${ }^{44}$ Any variation in site or field strengths within the sample leads to depolarization. The average lifetime of a muon is $2.2 \mu \mathrm{s}$, after which it decays into a positron, an antielectron neutrino and a muon neutrino. The positron is emitted preferentially in the direction of the muon polarization and is picked up by forward and backward detectors in $\pm \mathrm{z}$, where $\mathrm{z}$ is the muon beam direction. The $\mu \mathrm{SR}$ function $\mathrm{G}_{\mathrm{z}}(\mathrm{t})$ is extracted from the positron histograms in the forward and backward detectors, $\mathrm{N}_{\mathrm{F}}(\mathrm{t})$ and $\mathrm{N}_{\mathrm{B}}(\mathrm{t})$, by taking 
the ratio

$$
\begin{aligned}
\mathrm{P}(\mathrm{t}) & =\mathrm{a}_{\mathrm{o}} \mathrm{G}_{\mathrm{z}}(\mathrm{t}) \\
& =\left[N_{\mathrm{F}}(\mathrm{t})-\alpha \mathrm{N}_{\mathrm{B}}(\mathrm{t})\right] /\left[\mathrm{N}_{\mathrm{F}}(\mathrm{t})+\alpha \mathrm{N}_{\mathrm{B}}(\mathrm{t})\right],
\end{aligned}
$$

where $\alpha$ is an instrumental parameter to account for detector efficiencies and $\mathrm{a}_{\mathrm{o}}$ is the initial asymmetry, typically $0.21-$ 0.25. In the experiments on L20h and Harz, depolarization measurements were made in zero applied field and in longitudinal geometry.

On implantation into the goethite crystals, the muon, which behaves as a light hydrogen atom, naturally and rapidly diffuses to the location of a hydrogen ion vacancy in the lattice. It then encounters a local environment that, below $\mathrm{T}_{\mathrm{N}}$, is dominated by the large dipolar fields of the neighboring Fe atoms, and the muon precesses around that field, until the instant of disintegration. In the nonstoichiometric goethite, L20h, it is to be expected that there will be a significant siteto-site variation in the local field, while in the stoichiometric goethite, Harz, the local field will be more homogeneous. In both samples, and in keeping with the results from the TDR and ac susceptibility data described above, dynamic fluctuations in the direction of the local field are also to be anticipated, although we will not make any a priori assumptions as to what the timescales of those fluctuations might be.

Two sets of $\mu \mathrm{SR}$ data were recorded on the samples, at temperatures ranging from 10 to $425 \mathrm{~K}$. The first set (see Figs. 8 and 9) was recorded using the pulsed muon source at ISIS, which is well suited to probing the long-lifetime tails of $\mathrm{G}_{\mathrm{z}}(\mathrm{t})$, out to $\mathrm{t} \approx 10 \mu \mathrm{s}$, where the statistical quality of the data becomes poorer because fewer muons have persisted so long. The second set (see Figs. 10 and 11 and Table II) was recorded using the continuous source at Paul Scherrer
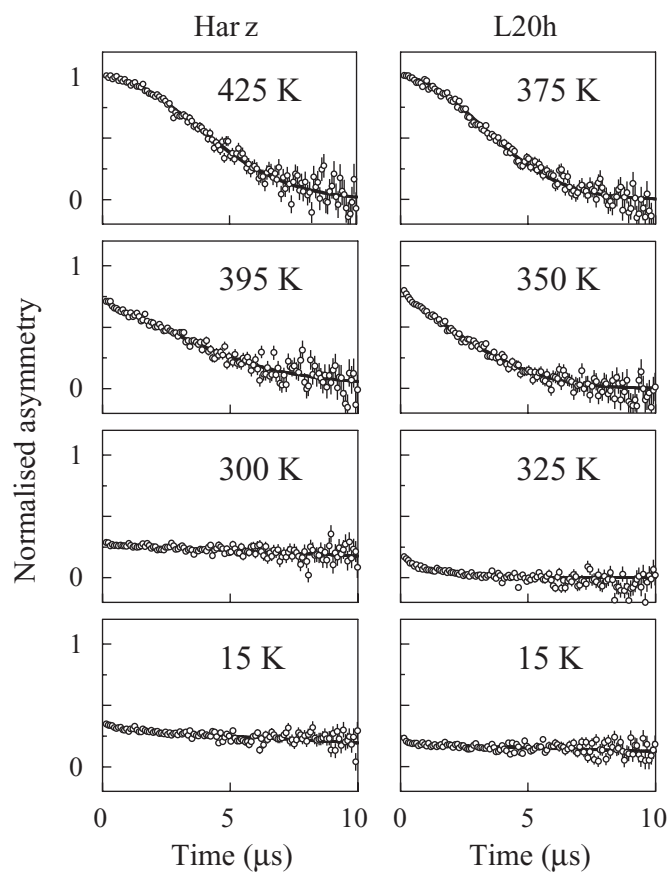

FIG. 8. Representative zero-field $\mu$ SR data for Harz and L20h goethite. The normalized function $\mathrm{G}_{\mathrm{z}}(\mathrm{t})$ is plotted, after correction for a background signal from a silver sample mount. Solid lines are least-squares fits using Eq. (6).

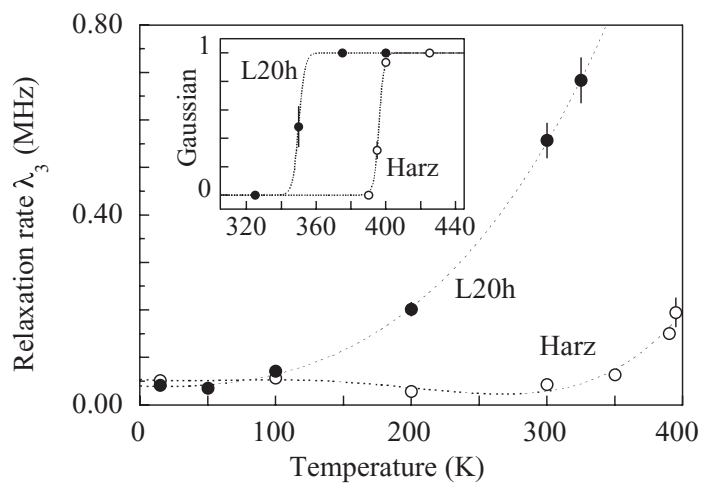

FIG. 9. Derived zero-field $\mu$ SR data for Harz and L20h goethite, fitted using Eq. (5). Dotted lines are guides to the eye. The main figure shows the relaxation rate $\lambda_{3}$ of the "slow" exponential component. The inset shows the relative amount of Gaussian component in the spectra, $a_{1} /\left(a_{0}-a_{f}\right)$, indicating the Néel transitions.

Institute (PSI), which afforded fast time resolution and very short dead times, making it possible to measure directly the rapid precession and depolarization of the muons in the first 200 ns after implantation.

The experimental data were fitted using a simple model

$$
\mathrm{P}(\mathrm{t})=\mathrm{a}_{1} \exp \left(-\mathrm{t}^{2} / \sigma_{1}^{2}\right)+\mathrm{a}_{\mathrm{f}}
$$

for $\mathrm{T} \geqslant \mathrm{T}_{\mathrm{N}}$, and a phenomenological model

$$
\mathrm{P}(\mathrm{t})=\sum_{\mathrm{i}} \mathrm{a}_{\mathrm{i}}\left[\frac{1}{3}+\frac{2}{3} \cos \left(\gamma_{\mu} \mathrm{B}_{\mathrm{i}} \mathrm{t}+\phi_{\mathrm{i}}\right)\right] \exp \left(-\lambda_{\mathrm{i}} \mathrm{t}\right)+\mathrm{a}_{\mathrm{f}}
$$

for $\mathrm{T}<\mathrm{T}_{\mathrm{N}}$. Equation (5) comprises the Gaussian form expected in the paramagnetic state, ${ }^{17,20}$ where the muon experiences only the static nuclear dipolar fields from its

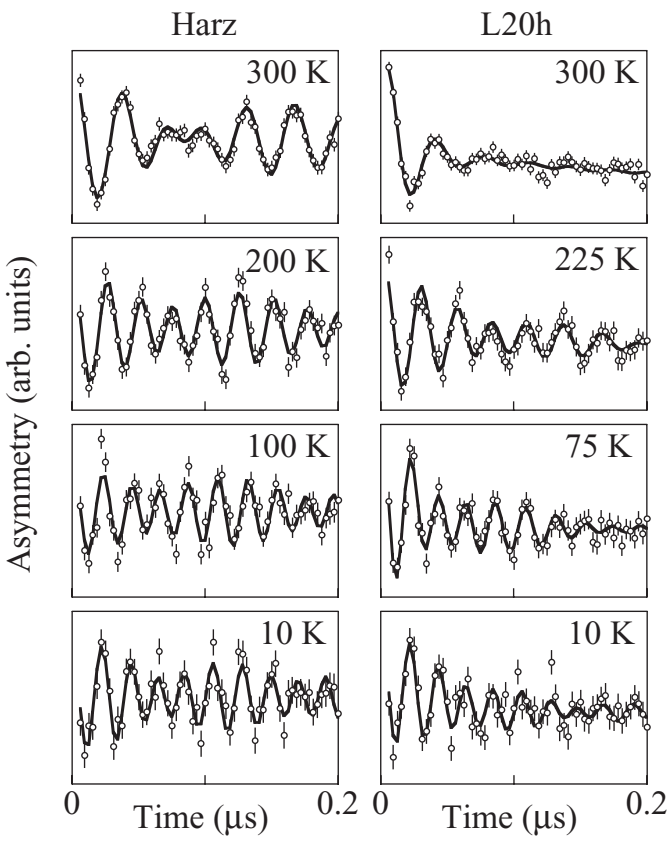

FIG. 10. High time resolution zero-field $\mu$ SR data for Harz and L20h goethite, highlighting the oscillatory signal due to the coherent precession of the implanted muons about the local filed axis. Solid lines are least-squares fits using Eq. (6) modified as in Eq. (7). 


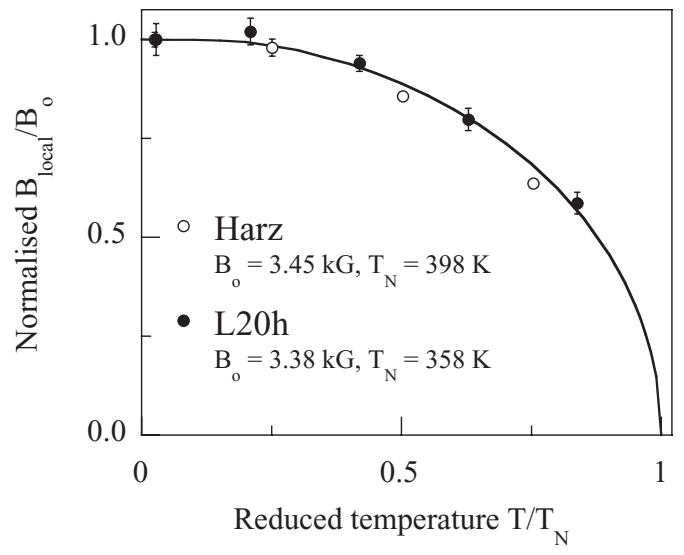

FIG. 11. Temperature dependence of the measured local field at a muon site determined from zero-field $\mu$ SR data for Harz and L20h goethite, compared to the Brillouin law curve for spin 5/2 (solid line).

neighboring hydrogen atoms, plus a constant offset $a_{f}$ due to the silver plate on which the samples were mounted.

Equation (6) represents the multitude of contributions to $\mathrm{P}(\mathrm{t})$ due to muons at sites with a local field of strength $\mathrm{B}_{\mathrm{i}}$, plus the offset $a_{f}$ due to the mounting plate. The field-dependent term is somewhat complex and needs some explanation. In principle, if all of the local fields in a magnetically ordered material took on only one value, $B_{0}$, then one would expect a precessional signal $\mathrm{G}_{\mathrm{z}}(\mathrm{t})=\cos \left(\gamma_{\mu} \mathrm{B}_{\mathrm{o}} \mathrm{t}+\varphi_{\mathrm{o}}\right)$, where $\gamma_{\mu}=$ $8.55 \mathrm{MHzG}^{-1}$ is the muon gyromagnetic ratio and $\varphi_{\mathrm{o}}$ is a phase angle. Note, however, that this would only be the case if the spin-polarized muon moment $\boldsymbol{\mu}$ were perpendicular to $\mathbf{B}_{\mathbf{0}}$. If instead $\boldsymbol{\mu}$ was parallel to $\mathbf{B}_{\mathbf{0}}$, there would be no precession at all, and $\mathrm{G}_{\mathrm{z}}(\mathrm{t})$ would be unity. In general it is the component of $\mathbf{B}_{\mathbf{o}}$ perpendicular to $\boldsymbol{\mu}$ that drives the precession. In a polycrystalline sample, such as the Harz and L20h samples, one should, therefore, expect a signal that is the weighted sum over a sphere. This, broadly speaking, accounts for the $\frac{1}{3}$ time-independent plus $\frac{2}{3}$ sinusoidally varying components to $G_{z}(t)$ in Eq. (6). However, even in this ideal scenario, the muons in different crystallites would precess at different rates governed by the angle between $\boldsymbol{\mu}$ and $\mathbf{B}_{\mathbf{0}}$ so that dephasing of the signal would occur. Again broadly speaking, such dephasing accounts for the exponential decay term $\exp \left(-\lambda_{\mathrm{i}} \mathrm{t}\right)$ in Eq. (6).
The situation in the antiferromagnetic Harz and L20h is, however, even more complicated. First, the magnitude of the local field, $B_{i}$ will be distributed about a mean value due to imperfections in the Fe lattice occupancy in both samples. This distribution will be broader in nonstochiometric L20h than in Harz, but in both cases it will contribute to a more rapid dephasing and depolarization of the signal. Second, there is the possible contribution of dynamic relaxation effects that could see the direction of the local field reversing at a time so close to the instant of disintegration that the muon would not have enough time to reach an equilibrium precessional state before disintegrating. Such effects would also lead to a drop in the asymmetry signal and could be difficult to distinguish experimentally from the polycrystalline sample and distributed local field effects, albeit that they might have a more pronounced temperature dependence. In any case, as seen below, Eq. (6) proved to be an adequate phenomenological model of the $\mu \mathrm{SR}$ function below $\mathrm{T}_{\mathrm{N}}$ for both sets of data (from ISIS and PSI), and with only two components, albeit that the interpretation of the meaning of the two components differs between the datasets.

The $\mu$ SR data in Figs. 8 and 10 clearly show that the dephasing of the sinusoidally varying part of the signal is rapid, occurring in the first $200 \mathrm{~ns}$ after implantation. As such, it is barely visible in the ISIS data, appearing only as a sharp exponential upturn at short $t$, while in the PSI data, it is clearly seen as a damped sinusoid. Consequently the ISIS data in Fig. 8 were best fitted as

$$
\begin{aligned}
\mathrm{P}(\mathrm{t})= & \mathrm{a}_{1} \exp \left(-\mathrm{t}^{2} / \sigma_{1}^{2}\right)+\frac{1}{3} \mathrm{a}_{2} \exp \left(-\lambda_{2} \mathrm{t}\right) \\
& +\frac{1}{3} \mathrm{a}_{3} \exp \left(-\lambda_{3} \mathrm{t}\right)+\mathrm{a}_{\mathrm{f}},
\end{aligned}
$$

which combines Eq. (5) and a two-term expansion of Eq. (6), and in which the sinusoidal component in the latter is assumed to have dephased to a zero net contribution. The total asymmetry parameter $\mathrm{a}_{\mathrm{o}}=\mathrm{a}_{1}+\frac{1}{3} \mathrm{a}_{2}+\frac{1}{3} \mathrm{a}_{3}+\mathrm{a}_{\mathrm{f}}$ was constrained to equal 0.24 at all temperatures.

For both samples at high temperatures, above ca. $350 \mathrm{~K}$ for L20h and ca. $396 \mathrm{~K}$ for Harz, the spectra took on the Gaussian form, indicating Néel transitions to the paramagnetic state (Fig. 9 inset). The fitted Gaussian standard deviations were $\sigma_{1} \approx 0.211 \mu \mathrm{s}^{-1}$ and $0.195 \mu \mathrm{s}^{-1}$ for L20h and Harz, respectively. Both values compare favorably with a theoretical estimate of $\sigma_{1} \approx 0.201 \mu \mathrm{s}^{-1}$ obtained from a nuclear dipole calculation, assuming muon implantation onto a hydrogen site

TABLE II. Parameters derived from the zero-field $\mu$ SR spectra of Fig. 9, fitted using Eqs. (6) and (7): the relative weighting of the two beating oscillations $\mathrm{a}_{2} /\left(\mathrm{a}_{2}+\mathrm{a}_{3}\right)$, the local field strengths $B_{2}$ and $B_{3}$, and the exponential relaxation rates $\lambda_{2}$ and $\lambda_{3}$. The number in

\begin{tabular}{|c|c|c|c|c|c|c|}
\hline Sample & Temperature (K) & $\mathrm{a}_{2} /\left(\mathrm{a}_{2}+\mathrm{a}_{3}\right)$ & $B_{2}(\mathrm{kG})$ & $\lambda_{2}(\mathrm{MHz})$ & $B_{3}(\mathrm{kG})$ & $\lambda_{3}(\mathrm{MHz})$ \\
\hline \multirow[t]{4}{*}{ Harz } & 300 & $0.51(5)$ & $1.77(1)$ & $5(1)$ & $2.23(1)$ & $3.1(3)$ \\
\hline & 200 & $0.28(8)$ & $2.35(2)$ & $5(2)$ & $2.95(1)$ & $2.9(3)$ \\
\hline & 100 & $0.22(6)$ & $2.77(1)$ & $2(1)$ & $3.38(1)$ & $2.4(4)$ \\
\hline & 10 & $0.34(12)$ & $2.76(4)$ & $7(3)$ & $3.49(1)$ & $2.8(5)$ \\
\hline \multirow[t]{4}{*}{ L20h } & 300 & $0.99(12)$ & $2.06(8)$ & $45(5)$ & $2.40(3)$ & $1(1)$ \\
\hline & 225 & $0.90(13)$ & $2.75(2)$ & $12(2)$ & $2.14(1)$ & $2(1)$ \\
\hline & 75 & $0.73(17)$ & $3.37(3)$ & $15(2)$ & $2.78(7)$ & $14(6)$ \\
\hline & 10 & $0.91(20)$ & $3.52(3)$ & $13(3)$ & $2.60(3)$ & $2(2)$ \\
\hline
\end{tabular}
parentheses is the uncertainty in the last digit. 
and neglecting the tiny contribution of the iron and oxygen nuclear dipoles. This is also confirmation that the muons implanted into goethite are diffusing to hydrogen lattice sites, as expected.

Below $\mathrm{T}_{\mathrm{N}}$ the Gaussian component fell to zero, and the data were fitted as two exponentials. One of these had a fast depolarization rate, $\lambda_{2} \approx 20-25 \mu \mathrm{s}^{-1}$, which did not vary significantly with temperature in either sample. The other had a slow depolarization rate, which was temperature dependent and different in the two samples. In Harz, $\lambda_{3}$ was close to zero until the sample temperature reached $300 \mathrm{~K}$, above which is showed a moderate rise to reach $\lambda_{3} \approx$ $0.2 \mu \mathrm{s}^{-1}$ at the Néel point. In L20h, $\lambda_{3}$ increased steadily with increasing temperature over the range $50-350 \mathrm{~K}$, reaching $\lambda_{3} \approx$ $0.7 \mu \mathrm{s}^{-1}$ at the Néel point (see Fig. 9). These observations are consistent with the previously identified cluster ordering relaxation in L20h giving rise to pronounced depolarization starting from low temperatures, while in Harz the increase in $\lambda_{3}$ as it approached its Néel temperature is consistent with more standard critical point dynamics.

More information on the fast relaxation component of the $\mu$ SR data was obtained from experiments undertaken using the continuous source at PSI (Fig. 10 and Table II). The fast time resolution and very short dead times available there made it possible to measure directly the rapid precession and depolarization in the first $200 \mathrm{~ns}$ after implantation. In this case the data were fitted using

$$
\begin{aligned}
\mathrm{P}(\mathrm{t})= & \frac{2}{3} \mathrm{a}_{2} \cos \left(\gamma_{\mu} \mathrm{B}_{2} \mathrm{t}+\phi_{2}\right) \exp \left(=\lambda_{2} \mathrm{t}\right) \\
& +\frac{2}{3} \mathrm{a}_{3} \cos \left(\gamma_{\mu} \mathrm{B}_{3} \mathrm{t}+\phi_{3}\right) \exp \left(-\lambda_{3} \mathrm{t}\right)+\mathrm{a}_{\mathrm{f}^{\prime}},
\end{aligned}
$$

which is a two-term expansion of Eq. (6), in which the $\frac{1}{3} a_{i}$ $\exp \left(-\lambda_{\mathrm{i}} \mathrm{t}\right)$ terms, which are not well defined over such a small time interval, have been treated as constants and incorporated into a single offset parameter, $\mathrm{a}_{\mathrm{f}^{\prime}}$.

It is clear from the data in Fig. 10 that the dephasing of the oscillatory $\mu \mathrm{SR}$ signal in L20h was faster than in Harz, as evidenced by the more rapid damping out of the sinusoidal oscillations in the signal. This is as expected given the broader distribution of local fields at the muon sites in the nonstoichiometric sample.

Furthermore, it is clear from the Harz data in Fig. 9 and Table II that two roughly equally weighted oscillatory signals are present, giving rise to a beatlike pattern. This is a significant observation since it could be due to the presence of two equally probable but slightly different Fe spins configurations in goethite, which underpins the prediction of MSPM relaxation. From the known magnetic and crystallographic structures of goethite, we can calculate the local field at the muon (hydrogen) lattice site. Following the data and nomenclature in Coey et al.'s neutron diffraction paper, ${ }^{18}$ we have calculated $\mathrm{B}_{\text {local }}$ at $10 \mathrm{~K}$ to be $3.81 \mathrm{kG}$ if the Fe moments are canted into the $C_{x}$ orientation and $3.90 \mathrm{kG}$ if they are canted in the $A_{z}$ orientation. The measured fields of ca. 2.8 and $3.5 \mathrm{kG}$ do not precisely fit with these predictions, although some reduction in the local field might be expected from imperfections in the lattice, and they are at least of the right order of magnitude. In support of this, recent high-resolution transmission electron microscopy images of goethite fine particles have provided direct evidence, through a study of lattice plane orientations, of modifications of the orthorhombic structure at intersections between neighboring crystallites. ${ }^{25}$

The observation of two distinct muon sites also has implications for the rate of MSPM fluctuations in Harz, which are evidently slower than the muon measurement. ${ }^{42}$ The predicted local field at the muon site if MSPM had been fast relative to $\tau_{m}$, with rapid fluctuation between $C_{x}$ and $A_{z}$ orientations leading to a time average zero of the off-axis component, is $\mathrm{B}_{\text {local }} \approx 2.80 \mathrm{kG}$ at $10 \mathrm{~K}$. The experimental observation of fields in excess of this value is only possible if the Fe moments are static on the $\mu$ SR measurement timescale.

Finally, the temperature dependence of the ordered state in both samples was probed by fitting the muon data to a single cosine-exponential term and comparing the resultant mean local field values against the expected Brillouin law dependence of a spin 5/2 antiferromagnet (Fig. 10). The good agreement between data and prediction indicates that the observed $\mu$ SR oscillations are indeed a true reflection of the intrinsic magnetic state of the samples.

\section{DISCUSSION}

In this study we set out to elucidate the intrinsic magnetic relaxation properties of goethite via a comparative study of stoichiometric and nonstoichiometric model samples. In goethite, there is a long-recognized correlation between stoichiometry and magnetic properties, most evident in a reduction in Néel temperature with increasing vacancy concentration. ${ }^{19}$ Recently, this correlation was codified by Barrero et al. ${ }^{41}$ into a magnetic phase diagram as a function of (1) the ratio of total water content to crystallite surface area, and (2) the Néel temperature. Mapped onto this phase diagram, Harz falls well within the antiferromagnetic region, while L20h lies near the boundaries of the antiferromagnetic and the weak ferromagnetism plus cluster ordering regions, ${ }^{41}$ validating our choice of samples. Incidentally, oxygen vacancies or weakly ferromagnetic impurities are common ingredients in the nature of goethite. ${ }^{45,46}$

The presence of MSPM, a never before seen but theoretically predicted phenomenon involving thermally induced fluctuations of the $\mathrm{Fe}$ moments over an energy barrier separating two closely related geometrical arrangements for the sublattice spins, ${ }^{18}$ was evidenced in TDR data. These data showed a power-exponential decay up to ca. $100 \mathrm{~K}$ in both Harz and L20h. Given the strong anisotropy of goethite and by persistent branching between the ZFC-FC $(\mathrm{H}=10 \mathrm{kOe})$ magnetization curves up to room temperature, this could not be attributed to full-moment or domain relaxation effects.

At intermediate timescales, samples by ac susceptometry served to verify the identification of MSPM in both samples and cluster ordering in L20h. Sharp upturns in the in-phase susceptibility $\chi^{\prime}(\mathrm{T})$ in both samples suggest that a mode fluctuation coupling (MSPM) is manifest. For high temperatures thermal fluctuations in the moment directions were sufficient to overcome the aligning effect of the time-varying 10-Oe applied field. In an extended set of frequency-dependent measurements on L20h, the low-temperature $\chi^{\prime}$ (T) upturn was observed at $\tau_{\mathrm{m}} \geqslant 330 \mu \mathrm{s}$ but was absent at $\tau_{\mathrm{m}}=100 \mu \mathrm{s}$, indicating that the latter was too fast for the Fe moments to follow. Corroborating evidence stemmed from the $\mu \mathrm{SR}$; the 
ca. 25-ns measurement time of the muons was fast enough to allow us to see, particularly in the Harz sample, where the confounding influence of distributed fields was minimal, two distinct sites for the implanted muons, with two distinguishable local fields. Given that MSPM depends on there being two closely related geometrical arrangements for the Fe sublattice spins, it should also be the case that if the measurement is fast enough, there should be two different local field values measured at the hydrogen sites in the lattice, which is where the muons end up. The fact that this was indeed seen, and that the measured local fields were at worst within $25 \%$ of the values predicted for a perfect lattice, is strong evidence in favor of the magnetic lattice canting effect, ${ }^{18}$ and by extension, supports the concept of MSPM. In this sense, the existence of the dynamic effects revealed here as MSPM may constitute a particular case among other systems displaying low-temperature spin dynamics as that, for instance, reported in horse-spleen ferritin $(<10 \mathrm{~nm})$, with two distinct distributions of anisotropy barriers, ${ }^{47}$ or another in Li-Ti ferrite spinels. ${ }^{48}$ Indeed, theoretical work has been reported to explain relaxation in noncollinear surface structures. ${ }^{49,50} \mathrm{It}$ is worth mentioning that the quantification of spin fluctuation modes should be of interest, but this would require further investigation via inelastic neutron scattering. ${ }^{51-53}$

Evidence for cluster ordering was seen in the nonstoichiometric L20h sample only, in the form of a peak in the ZFC magnetization curves. The temperature at which the peak occurred ranged from $40 \mathrm{~K}$ in a field $\mathrm{H}=10 \mathrm{kOe}$ to $70 \mathrm{~K}$ in $\mathrm{H}=10 \mathrm{Oe}$ and was found to vary with the field as observed in glassy and/or strongly interacting magnetic states. ${ }^{37,38}$ Cluster ordering in L20h was evidenced by frequency-dependent peaks in $\chi^{\prime}(\mathrm{T})$ at ca. $60-70 \mathrm{~K}$, whose functional dependence followed a Vogel-Fulcher law, typical of ordering in interacting systems. ${ }^{39,40}$ There are alternative explanations: in particular, the observation of frequency-dependent $\chi^{\prime}(\mathrm{T})$ peaks could be taken as a sign of superferromagnetism, as recently discussed in hematite and Fe-Ag fims. 5,6,54 The two interpretations, cluster ordering and superferromagnetism, are closely related, differing in the length scale of the interacting coherently magnetized regions: clusters and particles, respectively. However, with the data at hand, following the standard rule of thumb $b^{39}$ that relates the volume $V$ of a coherently magnetized entity to the zero-field superparamagnetic blocking temperature $\mathrm{T}_{\mathrm{B}}$ for that entity, $V \approx 25 \mathrm{kT}_{\mathrm{B}} / K$, where $K=45.7 \mathrm{kJm}^{-3}$ is the magnetic anisotropy energy of goethite, ${ }^{27}$ we obtain, for $\mathrm{L} 20 \mathrm{~h}$ with its $\chi^{\prime}(\mathrm{T})$ peak at ca. $70 \mathrm{~K}$, the estimate that $V \approx 530 \mathrm{~nm}^{3}$. As this is much smaller than the ca. $3590 \mathrm{~nm}^{3}$ known volume of the particles in L20h, it appears that magnetic clusters are forming in the crystallites and that cluster ordering is the most likely origin of the observed relaxation.

Furthermore, the muon results show in L20h that an almost monotonically increasing slow relaxation component was seen from $50-350 \mathrm{~K}$ in data recorded at ISIS on the statistically small fraction of muons that disintegrated up to $10 \mu \mathrm{s}$ after implantation. The equivalent effect was not seen in the ISIS data on the Harz sample, leading us to attribute it to cluster ordering.

\section{CONCLUSIONS}

Through a series of experiments on both stoichiometric and nonstoichiometric goethite, it is plausible that the predicted magnetic relaxation mechanism so-called "mode superparamagnetism" is an intrinsic feature of the mineral. This dynamic phenomenon, in which the $\mathrm{Fe}$ moments in the material undergo thermally induced fluctuations over an energy barrier separating two closely related geometrical arrangements for the sublattice spins, was experimentally observed here via ac susceptibility and TDR measurements. At measurement times shorter than ca. $100 \mu \mathrm{s}$, this relaxation mode was not observed, and in both $\mu \mathrm{SR}$ and Mössbauer spectra, the Fe moments were static. In the muon data, two distinct local magnetic fields were measured and found to be due to the two Fe lattice configurations that constitute the MSPM ground states.

An additional magnetic relaxation mode was measured in the nonstoichiometric goethite only and was identified as being due to "cluster ordering." This mode appeared in the ac susceptibility and dc magnetization data as blocking transitions (peaks) in the range 70 to $100 \mathrm{~K}$ that, respectively, followed Vogel-Fulcher-like frequency laws and were strongly affected by the applied field. Both are indications of cluster (glassy) ordering: the formation and relaxation of coherently magnetized magnetic clusters in the disturbed environment of the nonstoichiometric lattice.

In conclusion, it is intriguing to note that it is more than 40 years since researchers first began speculating on the static and dynamic magnetic properties of what at first appeared to be an extremely simple mineral: goethite. Yet, as this study (and the many that have gone before) has demonstrated, the intrinsic magnetic relaxation properties are somewhat difficult to discern, albeit straightforward enough to understand once one has accumulated (1) sufficient data of (2) requisite quality at (3) an appropriate range of measurement timescales. We can learn from this. As we look ahead to a future in which new magnetically heterogeneous materials become more and more technologically important, in fields as diverse as magnetic recording and biomagnetism, the need for methods whereby we can analyze and interpret complex nanosystems is likely to also become even more important. We hope that the methods exemplified here may act as a starting point for such studies to come.

\section{ACKNOWLEDGMENTS}

We thank Steve Cox (ex RAL), Ivan Reid (ex PSI), Stephen Bocquet (ex Monash University), and Eddy de Grave (Ghent) for their help, and the Spanish CICYT for their support via grant Projects No. MAT2008-06542-C04-04 and No. MAT2011-27573-C04-01. *qpankhurst@ri.ac.uk

${ }^{1}$ J. F. Banfield, S. A. Welch, H. Z. Zhang, T. T. Ebert, and R. L. Penn, Science 289, 751 (2000).

${ }^{2}$ H. Ohmoto, Y. Watanabe, and K. Kumazawa, Nature 429, 395 (2004).
${ }^{3}$ M. L. Wells, L. M. Mayer, O. F. X. Donard, M. M. D. Sierra, and

S. G. Ackelson, Nature 353, 248 (1991).

${ }^{4}$ J. Dobson, FEBS Lett. 496, 1 (2001).

${ }^{5}$ C. Binns, M. J. Maher, Q. A. Pankhurst, D. Kechrakos, and K. N. Trohidou, Phys. Rev. B 66, 184413 (2002). 
${ }^{6}$ S. Bedanta and W. Kleemann, J. Phys. D 42, 013001 (2009); S. Bedanta, T. Eimüller,W. Kleemann, J. Rhensius, F. Stromberg, E. Amaladass, S. Cardoso, and P. P. Freitas, Phys. Rev. Lett. 98, 176601 (2007).

${ }^{7}$ V. Skumryev, S. Stoyanov, Y. Zhang, G. Hadjipanayis, D. Givord, and J. Nogues, Nature 423, 850 (2003).

${ }^{8}$ M. F. Hansen, C. B. Koch, and S. Mørup, Phys. Rev. B 62, 1124 (2000).

${ }^{9}$ J. M. De Teresa, M. R. Ibarra, J. García, J. Blasco, C. Ritter, P. A. Algarabel, C. Marquina, and A. del Moral, Phys. Rev. Lett. 76, 3392 (1996).

${ }^{10}$ J. M. De Teresa, M. R. Ibarra, P. Algarabel, L. Morellon, B. GarcíaLanda, C. Marquina, C. Ritter, A. Maignan, C. Martin, B. Raveau, A. Kurbakov, and V. Trounov, Phys. Rev. B 65, 100403 (2002).

${ }^{11}$ J. Nogués, J. Sort, V. Langlais, V. Skumryev, S. Suriñach, J. S. Muñoz, and M. D. Baró, Phys. Rep. 422, 65 (2005).

${ }^{12}$ O. Iglesias, A. Labarta, and X. Batlle, J. Nanosci. Nanotechnol. 8, 2761 (2008).

${ }^{13}$ S. Mørup, D. E. Madsen, C. Frandsen, C. R. H. Bahl, and M. F. Hansen, J. Phys.: Condens. Matter 19, 213202 (2007).

${ }^{14}$ D. W. Strangway, R. M. Honea, B. E. McMahon, and E. E. Larson, Geophys. J. R. Astron. Soc. 15, 345 (1968).

${ }^{15}$ P. Rochette and G. Fillion, Geophys. Res. Lett. 16, 851 (1989).

${ }^{16}$ I. G. Hedley, Z. Geophysik 37, 409 (1971).

${ }^{17}$ D. Broz and B. Sedlak, J. Magn. Magn. Mater. 102, 103 (1991).

${ }^{18}$ J. M. D. Coey, A. Barry, J. M. Brotto, H. Rakoto, S. Brennan, W. N. Mussel, A. Collomb, and D. Fruchart, J. Phys.: Condens. Matter 7, 759 (1995).

${ }^{19}$ F. Van der Woude and A. J. Dekker, Phys. Status Solidi 13, 181 (1966).

${ }^{20}$ J. B. Forsyth, I. G. Hedley, and C. E. Johnson, J. Phys. C 1, 179 (1968).

${ }^{21}$ A. M. Van der Kraan and J. J. Van Loef, Phys. Lett. 20, 614 (1966).

${ }^{22}$ T. Shinjo, J. Phys. Soc. Jpn. 21, 917 (1966).

${ }^{23}$ S. Mørup, M. B. Madsen, J. Franck, J. Villadsen, and C. J. W. Koch, J. Magn. Magn. Mater. 40, 163 (1983).

${ }^{24}$ D. E. Madsen, M. F. Hansen, C. B. Koch, and S. Mørup, J. Phys.: Condens. Matter 20, 135215 (2008).

${ }^{25}$ D. E. Madsen, L. Cervera-Gontard, T. Kasama, R. E. DuninBorkowski, C. B. Koch, M. F. Hansen, C. Frandsen, and S. Mørup, J. Phys.: Condens. Matter 21, 016007 (2009).

${ }^{26}$ S. Bocquet and S. J. Kennedy, J. Magn. Magn. Mater. 109, 260 (1992).

${ }^{27}$ S. Bocquet, R. J. Pollard, and J. D. Cashion, Phys. Rev. B 46, 11657 (1992)

${ }^{28}$ S. Bocquet, J. Phys.: Condens. Matter 8, 111 (1996).

${ }^{29}$ F. L. Pratt, Physica B 289, 710 (2000).

${ }^{30}$ E. De Grave and R. E. Vandenberghe, Hyperfine Interact. 28, 643 (1986).
${ }^{31}$ E. Murad and J. H. Johnston, in Mössbauer Spectroscopy Applied to Inorganic Chemistry, edited by G. J. Long, Vol. 2 (Plenum Press, New York, 1987), pp. 507-582.

${ }^{32}$ S. Bocquet and A. J. Hill, Phys. Chem. Miner. 22, 524 (1995).

${ }^{33}$ A. B. Harris and S. Kirkpatrick, Phys. Rev. B 16, 542 (1977).

${ }^{34}$ D. G. Schulze and U. Schwertmann, Clay Miner. 19, 521 (1984).

${ }^{35}$ Q. A. Pankhurst, Hyperfine Interact. 90, 201 (1994).

${ }^{36}$ J. L. Dormann, D. Fiorani, and E. Tronc, Adv. Chem. Phys. 98, 283 (1997).

${ }^{37}$ B. Martínez, X. Obradors, L. Balcells, A. Rouanet, and C. Monty, Phys. Rev. Lett. 80, 181 (1998).

${ }^{38}$ J. L. Dormann, D. Fiorani, and M. Elyamani, Phys. Lett. A 120, 95 (1987).

${ }^{39}$ T. S. Berquó, R. A. L. Imbernon, A. Blot, D. R. Franco, M. C. M. Toledo, and C. S. M. Partiti, Phys. Chem. Miner. 34, 287 (2007).

${ }^{40}$ J. L. Dormann, L. Bessais, and D. Fiorani, J. Phys. C 21, 2015 (1988).

${ }^{41}$ C. A. Barrero, J. D. Betancur, J. M. Greneche, G. F. Goya, and T. S. Berquó, Geophys. J. Int. 164, 331 (2006).

${ }^{42}$ The characteristic timescale $\tau_{\mathrm{m}}$ of $\mu \mathrm{SR}$ is sample dependent, as it depends on the muon having achieved an equilibrium precession about the local field B before it spontaneously decays and releases a positron that is subsequently detected. This, in turn, depends on the magnitude of $B$. In the case that $B=1 \mathrm{kG}$, we estimate $\tau \mathrm{m}$ to be the time required for the muon to precess 100 times, which is $100 \times 2 \pi / \gamma \mu \mathrm{B} \approx 70 \mathrm{~ns}$. If $\mathrm{B}=3 \mathrm{kG}, \tau_{\mathrm{m}} \approx 25 \mathrm{~ns}$.

${ }^{43}$ Leila Rebbouh, Raphaël P. Hermann, Fernande Grandjean, Taeghwan Hyeon, Kwangjin An, Alex Amato, and Gary J. Long, Phys. Rev. B 76, 174422 (2007).

${ }^{44}$ S. F. J. Cox, J. Phys. C 20, 3187 (1987).

${ }^{45}$ J. Majzlan, A. Navrotsky, B. F. Woodfield, B. E. Lang, J. BoerioGoates, and R. A. Fisher, J. Low Temp. Phys. 130, 69 (2003).

${ }^{46}$ F. Martín-Hernandez and M. García-Hernández, Geophys. J. Int. 181, 756 (2010)

${ }^{47}$ T. G. St. Pierre, N. T. Gorham, P. D. Allen, J. L. Costa-Krämer, and K. V. Rao, Phys. Rev. B 65, 024436 (2001).

${ }^{48}$ J. L. Dormann, M. El Harfaouit, M. Noguès, and J. Jove, J. Phys. C 20, L161 (1987).

${ }^{49}$ R. H. Kodama, A. E. Berkowitz, E. J. McNiff Jr., and S. Foner, Phys. Rev. Lett. 77, 394 (1996).

${ }^{50}$ S. Mørup, J. Magn. Magn. Mater. 266, 110 (2003).

${ }^{51}$ M. J. Harris, M. P. Zinkin, and T. Zeiske, Phys. Rev. B 52, R707 (1995).

${ }^{52}$ U. Falk, A. Furrer, N. Furer, H. U. Güdel, and J. K. Kjems, Phys. Rev. B 35, 4893 (1987).

${ }^{53}$ M. F. Hansen, F. Bødker, S. Mørup, K. Lefmann, K. N. Clausen, and P.-A. Lindgård, Phys. Rev. Lett. 79, 4910 (1997).

${ }^{54}$ J. Alonso, M. L. Fdez-Gubieda, J. M. Barandiarán, A. Svalov, L. Fernández Barquín, D. Alba Venero, and I. Orue, Phys. Rev. B 82, 054406 (2010). 